\title{
Effects of Vegan and Ketogenic Diet Plans on Hemoglobin A1c Levels in Type 2 Diabetes
}

\author{
Kimberly Jones ${ }^{1}$, Erin Jefferson ${ }^{2}$, Brian Henriksen ${ }^{1,2}$ \\ ${ }^{1}$ Indiana University School of Medicine; ${ }^{2}$ Fort Wayne Medical Education Program, Department \\ of Family Medicine
}

Background/Objective: Type 2 diabetes affects $8.5 \%$ of adults over the age of 18 and costs the United States an average of 327 billion dollars per year. Two diets, the ketogenic diet and the vegan diet have both been shown to promote weight loss as well as reduce A1c's in individuals with type 2 diabetes. Ketogenic diets are best defined as high fat, low carbohydrate diets that induce a state of nutritional ketosis. Vegan diets are centered around the consumption of legumes, fruits, vegetables, and whole grains. All animal-derived products including gelatin are avoided. The primary goal of this study is to determine whether the vegan diet is non-inferior to the ketogenic diet in the reduction of hemoglobin A1c levels in adults with type 2 diabetes.

Methods: A total of 120 participants between the ages of 18 and 70 who have an A1c between 6.5 and 10.0 will randomly be assigned to either the vegan or ketogenic diet groups. Dietary interventions will be implemented for a total of 24 weeks. Hemoglobin A1c levels will be measured at weeks 0,12 , and 24 and values will be compared using the repeated-measures analysis of variance test to determine if there is a significant difference between groups.

Expected results: It is expected that both groups will experience a decrease in their hemoglobin A1c levels, but that a more significant reduction will occur in those following the vegan diet. Weight loss is also an anticipated side effect in both groups.

Conclusion/Potential impact: This study has the potential to provide individuals with a more cost-effective management strategy to medication therapy in type 2 diabetes. Choosing to adopt healthier dietary practices will ultimately lead to better blood glucose control and a lower likelihood of developing diabetic complications. 